\title{
Pelvic Ultrasound Findings in Women with Obstetric Fistula: A Cross-Sectional Study of Cases and Controls
}

\author{
Jeffrey P. Wilkinson $\mathbb{D}^{1},{ }^{1}$ Angela M. Bengtson, ${ }^{2}$ Ennet Chipungu, ${ }^{3}$ Rachel J. Pope $\mathbb{D}^{1}{ }^{1}$ \\ Bonus Makanani, ${ }^{4}$ Margaret Moyo, ${ }^{3}$ Mwawi Mwale, ${ }^{5}$ and Jennifer H. Tang $\mathbb{D}^{6}$ \\ ${ }^{1}$ Baylor College of Medicine, Department of Obstetrics and Gynecology, Global Women's Health, Baylor Plaza, \\ Houston, TX 77030, USA \\ ${ }^{2}$ University of North Carolina, Department of Epidemiology, 401 Rosenau Hall, Chapel Hill, NC 27599-7455, USA \\ ${ }^{3}$ Freedom from Fistula Foundation, Fistula Care Centre, Lilongwe, Malawi \\ ${ }^{4}$ Department of Obstetrics and Gynecology, Malawi College of Medicine, Blantyre, Malawi \\ ${ }^{5}$ Malawi Ministry of Health, Bwaila Maternity Hospital, Lilongwe, Malawi \\ ${ }^{6}$ University of North Carolina, Department of Obstetrics and Gynecology, 101 Manning Drive, Chapel Hill, NC 27599-7570, USA
}

Correspondence should be addressed to Jeffrey P. Wilkinson; jeffrey.wilkinson@bcm.edu

Received 1 June 2017; Accepted 19 September 2017; Published 10 January 2018

Academic Editor: Peter E. Schwartz

Copyright (c) 2018 Jeffrey P. Wilkinson et al. This is an open access article distributed under the Creative Commons Attribution License, which permits unrestricted use, distribution, and reproduction in any medium, provided the original work is properly cited.

\begin{abstract}
Objective. Obstetric fistula (OF) is a morbid condition caused by prolonged obstructed labor. Women with OF experience profound injury and have high rates of infertility and poor obstetric outcomes. We examined endovaginal ultrasound parameters in women with and without OF. Design/Setting/Sample/Methods. This cross-sectional study enrolled women evaluated at the Fistula Care Centre in Lilongwe, Malawi. Eligibility criteria included age 18-45, prior pregnancy, and a uterus on ultrasound. Participants underwent endovaginal ultrasound with measurement of cervical dimensions. Comparisons were done using $t$-tests and Fisher's exact test. Among women with OF, linear regression was used to assess whether fistula stage was associated with cervical length. Results. We enrolled 98 cases and 12 controls. Women with OF had shorter cervical lengths $(18.8 \mathrm{~mm}$ versus $27.3 \mathrm{~mm}, p<0.01)$, as well as shorter anterior $(7.0 \mathrm{~mm}$ versus $9.3 \mathrm{~mm}, p<0.01)$ and posterior $(9.5 \mathrm{~mm}$ versus $11.0 \mathrm{~mm}, p<0.04)$ cervical stroma, compared to controls. Conclusion. Women with OF have shorter cervical lengths and anterior and posterior cervical stroma, when compared to women without OF. This may offer a partial explanation for subfertility and poor obstetric outcomes in OF patients. Additional studies to clarify the role of ultrasound in OF patients and prediction of future fertility are warranted.
\end{abstract}

\section{Introduction}

Obstetric fistula $(\mathrm{OF})$ is a devastating condition that occurs when women experience prolonged obstructed labor. The soft tissues of the pelvis undergo tissue necrosis, resulting in an abnormal communication between the vagina and the bladder and/or rectum. Women with OF continually leak urine and/or feces from their vagina until they are surgically repaired [1].

Many women with OF develop secondary infertility or amenorrhea, although some become pregnant again, before or after surgical repair. For those who do become pregnant, their pregnancies are often associated with high rates of preterm birth and spontaneous abortion, particularly in the second trimester [2-4]. The reasons for this are not well understood, although experts have hypothesized that women with OF have higher rates of preterm birth because of severe cervical damage and avulsion that occur during prolonged obstructed labor, which leads to cervical insufficiency [5]. In extreme cases, fistula surgeons have noted that patients have no identifiable cervical tissue $[1,6,7]$. A case series of $22 \mathrm{OF}$ patients found that, on vaginal exam, $40 \%$ of patients had a normal cervix, $23 \%$ had cervical erosion, $18 \%$ had only a cervical dimple, $14 \%$ had a nonidentifiable cervix (because it 
was bound to the pubic symphysis), and 5\% had a "harelip" defect of the anterior cervix. On transabdominal ultrasound, $68 \%$ had a normal-appearing cervix and $9 \%$ had anterior cervical defects [7]. No published study has assessed the use of endovaginal ultrasound to evaluate the cervix in OF patients.

Cervical length measurement is often performed during pregnancy to assess for short cervix, which is associated with preterm birth [8]. Women found to have a short cervix due to structural weakness of the cervix, as in the case of prior cone biopsy or a Müllerian duct anomaly, may benefit from placement of a prophylactic cerclage [9-11]. No published studies have examined the use of prepregnancy cervical length as a predictor for preterm birth in women at risk for having a short or abnormal cervix. The cervix lengthens during the first trimester of pregnancy, so pregnant women generally have longer cervices than nonpregnant women $[12,13]$. The prepregnancy cervical length may have some predictive value, particularly in women with prior cervical trauma or surgery. For OF patients, pregnancy is uncommon, and they interface with the health care system infrequently. It is more practical to assess their pelvic anatomy by ultrasound at the time of their repair. If $\mathrm{OF}$ patients are found to have compromised pelvic anatomy, this could result in counseling on future pregnancy risk or potential interventions to prevent early pregnancy loss or other complications.

Therefore, we conducted this study to evaluate whether women with OF have a shorter prepregnancy cervical length than women without OF when measured by endovaginal ultrasound. We hypothesized that women with OF would have a significantly shorter cervical length when compared to women without $\mathrm{OF}$.

\section{Methods}

This study was conducted at a 40-bed hospital unit dedicated to the care of obstetric fistula patients located on the grounds of the Bwaila Maternity Hospital in Lilongwe, Malawi. Between December 2012 and June 2014 (when funding for this study ended), a convenience sample of women who presented for surgical care of OF (defined as vesicovaginal fistula and/or rectovaginal fistula that developed during or immediately after labor) were recruited for participation as cases. Women who presented for evaluation of other gynecologic conditions (such as stress urinary incontinence or anal sphincter tears during childbirth) who did not have OF or a history of prolonged obstructed labor were recruited as controls. Inclusion criteria included women able to provide consent in Chichewa, aged 18-45 years, and a willingness to undergo the ultrasound examination at the time of surgery or during pelvic examination. Exclusion criteria included nulliparity, history of hysterectomy, current pregnancy, or presence of serious or terminal illness, such as advanced cervical cancer.

The study was approved by the Malawi National Health Sciences Research Committee, as well as the University of North Carolina Institutional Review Board/Office of Human Research Subjects. Prior to surgery in the cases or prior to examination in the controls, informed consent was obtained in writing after explanation by a native Chichewa speaker.

In study participants with $\mathrm{OF}$, endovaginal ultrasound was performed after spinal anesthesia was administered for surgery. Most women had an empty bladder because of their $\mathrm{OF}$, but in a few cases, there was sufficient urine to require catheterization prior to ultrasound. Endovaginal ultrasound was performed on all participants, by the same sonographer in lithotomy position. A systematic ultrasound examination was performed which measured the cervical length three times. The final cervical length was defined as the shortest and best of the three measurements. This was followed by measurement of cervical width, anterior and posterior cervical stroma, and uterine volume (length, width, and depth). Uterine position was assessed, and endometrial thickness, the percentage of the endometrium visualized, endometrial appearance and echogenicity, and other endometrial findings were noted. The presence or absence of fibroids including number, position, and size were also documented. Visualization of the ovaries was noted with ovarian volume measurements, presence of follicles, cysts, or other masses. Final uterine volume was calculated with the formula length $\times$ width $\times$ depth $\times 4 / 3 \times \pi$, and ovarian volume was calculated with the formula length $\times$ width $\times$ depth $\times \pi / 6$. The examiner noted his clinical impression of the likely cycle phase as part of the examination, based on the appearance of the endometrium and ovarian follicular development. The ovarian follicle data are published separately as part of an analysis of amenorrhea and hormone levels in OF patients [2].

Controls underwent endovaginal ultrasound in the lithotomy position during a pelvic examination after they had emptied their bladder. The same systematic approach to the ultrasound examination and measurements was performed.

Data were collected in a form designed for this study and then double entered and cleaned in a secure Research Electronic Data Capture (REDCap) study database [14], which is both web-based and password-protected. The REDCap database was managed by the North Carolina Translational and Clinical Sciences (NC TraCS) Institute and stored on a secure UNC server. The REDCap data were exported and then analyzed using STATA (StataCorp, College Station, TX).

\section{Data Analysis}

We compared demographic and clinical characteristics between cases with OF and controls without OF using Fisher's exact test. Cervical, endometrial, uterine, and ovarian characteristics were characterized for all women using descriptive statistics and compared between cases and controls using $t$-tests for continuous variables, the MannWhitney $U$ test for ordinal variables, and Fisher's exact test for categorical variables. Among cases with OF who had a final cervical length measurement, we used linear regression to calculate mean differences and $95 \%$ confidence intervals in final cervical length by both major and minor Waaldijk classifications. All statistical analyses were done in Stata version 13 (StataCorp, College Station, Texas). 
TABLE 1: Demographic and clinical characteristics of 110 Malawian women seeking care at an obstetric fistula center in Lilongwe, Malawi, between December 2012 and June 2014.

\begin{tabular}{|c|c|c|c|}
\hline Variable & $\begin{array}{c}\text { Obstetric fistula }(N=98) \\
N(\%)\end{array}$ & $\begin{array}{c}\text { No obstetric fistula }(n=12) \\
\qquad N(\%)\end{array}$ & $p$ value $^{\mathrm{a}}$ \\
\hline Age & & & 0.57 \\
\hline $18-24$ & $21(21.4)$ & $2(16.7)$ & - \\
\hline $25-34$ & $47(48.0)$ & $8(66.7)$ & - \\
\hline $35-45$ & $30(30.6)$ & $2(16.7)$ & - \\
\hline Parity & & & 0.37 \\
\hline 1 & $27(27.6)$ & $1(8.3)$ & - \\
\hline $2-3$ & $38(38.8)$ & $6(50.0)$ & - \\
\hline$\geq 4$ & $33(33.7)$ & $5(41.7)$ & - \\
\hline $\mathrm{BMI}^{\mathrm{b}}$ & & & 0.81 \\
\hline$<18.5$ & $8(9.0)$ & $2(18.2)$ & - \\
\hline $18.5-<25.0$ & $54(71.9)$ & $8(72.7)$ & - \\
\hline $25.0-<30.0$ & $11(12.4)$ & $1(9.1)$ & - \\
\hline$\geq 30$ & $6(6.7)$ & $0(0.0)$ & - \\
\hline Marital status & & & 0.17 \\
\hline Not married & $31(31.6)$ & $1(8.3)$ & - \\
\hline Married & $67(68.4)$ & $11(91.7)$ & - \\
\hline Education & & & 0.08 \\
\hline None or primary & $91(92.9)$ & $9(75.0)$ & - \\
\hline Secondary or more & $7(7.1)$ & $3(25.0)$ & - \\
\hline Occupation & & & 0.34 \\
\hline Other $^{c}$ & $28(28.6)$ & $5(41.7)$ & - \\
\hline Peasant farmer & $70(71.4)$ & $7(58.3)$ & - \\
\hline HIV status & & & 0.24 \\
\hline Positive & $5(5.1)$ & $2(16.7)$ & - \\
\hline Negative & $84(85.7)$ & $9(75.0)$ & - \\
\hline Don't know & $9(9.2)$ & $1(8.3)$ & - \\
\hline
\end{tabular}

${ }^{\mathrm{a}}$ Fisher's exact test; ${ }^{\mathrm{b}} \mathrm{BMI}$ : body mass index, calculated as $\mathrm{kg} / \mathrm{m}^{2}$; “"Other”: unemployed, housewife or caretaker, large or small business owner, casual laborer, other, don't know. Missing data: BMI $n=10$.

\section{Results}

A total of 110 women were included in the study: 98 cases with $\mathrm{OF}$ and 12 controls without OF. Both cases and controls were most likely to be 25-34 years of age, to have given birth to 2-3 children, be HIV-uninfected, and have a normal body mass index (18.5-25.0). While not statistically different, women with OF were more likely to not be married (32\% versus $8 \%$ ), to have not gone to school or only have gone to primary school $(93 \%$ versus $75 \%$ ), and to be a peasant farmer (71\% versus $58 \%$ ), compared to women without OF (Table 1).

The cervix could be visualized and measured in 90 of the 98 women with OF and all women without OF. Women with OF were found to have significantly shorter cervical lengths than women without OF, after adjustment for parity $(18.8 \mathrm{~mm}$ versus $27.3 \mathrm{~mm}, p$ value $<0.01)$. Both anterior and posterior cervical stroma were shorter in women with $\mathrm{OF}$ than controls $(7.0 \mathrm{~mm}$ versus $9.3 \mathrm{~mm}, p$ value 0.01 , and $9.5 \mathrm{~mm}$ versus $11 \mathrm{~mm}, p$ value 0.04 , resp.). There were no significant differences in cervical width, endometrial thickness, or uterine volume between cases and controls. The endometrium was fully visualized in $78 \%$ of cases and $100 \%$ of controls ( $p$ value 0.07 ).
No significant differences were noted in other endometrial or ovarian characteristics (Table 2).

Of the 98 women included with obstetric fistula, 90 had a final cervical length measured (92\%). Overall, the largest proportion of women had a fistula categorized as Waaldijk Major IIAb/IIBb $(n=40,44 \%)$ or Waaldijk Minor large/extensive $(n=30,33 \%)$. Women with more severe fistula classifications had slightly shorter final cervical lengths on average. However, there were no statistically significant associations between stage of the fistula as determined by the Waaldijk classification system and cervical length (Table 3).

\section{Discussion}

This cross-sectional endovaginal ultrasound study compared women with OF caused by prolonged obstructed labor to women without OF or prolonged obstructed labor. Women with OF had significantly shorter cervical lengths and anterior cervical stroma. This is the first study to systematically examine the endovaginal ultrasound characteristics of the cervix and other pelvic organs in women with OF. Often, one is unable to visualize or palpate the 
TABle 2: Differences in obstetric characteristics by obstetric fistula status, among 110 women in Malawi.

\begin{tabular}{|c|c|c|c|c|}
\hline Variable & $\begin{array}{c}\text { Obstetric fistula }(N=98) \\
\text { Mean }(\mathrm{SD}) \text { or } N(\%)\end{array}$ & $\begin{array}{c}\text { No obstetric fistula }(n=12) \\
\text { Mean }(\mathrm{SD}) \text { or } N(\%)\end{array}$ & $p$ value & Missing \\
\hline Final cervical length, $\mathrm{mm}$ & $18.8(8.7)$ & $27.3(6.2)$ & $<0.01$ & 8 \\
\hline Cervical width, mm & $18.5(8.0)$ & $21.45(3.0)$ & 0.21 & 8 \\
\hline Anterior cervical stroma, mm & $7.0(3.1)$ & $9.3(1.4)$ & 0.01 & 10 \\
\hline Posterior cervical stroma, $\mathrm{mm}$ & $9.5(2.5)$ & $11.0(1.5)$ & 0.04 & 9 \\
\hline Final uterine volume, $\mathrm{cm}^{3}$ & $587.7(279.8)$ & $628.2(303.3)$ & 0.64 & 0 \\
\hline Endometrial thickness, $\mathrm{mm}$ & $7.0(5.1)$ & $7.1(5.2)$ & 0.96 & 0 \\
\hline Endometrium visualized & & & 0.07 & 0 \\
\hline $100 \%$ & $76(77.6)$ & $12(100.0)$ & - & - \\
\hline $51-99 \%$ & $12(12.2)$ & $0(0.0)$ & - & - \\
\hline$\leq 50 \%$ & $9(9.2)$ & $0(0.0)$ & - & - \\
\hline $0 \%$ & $1(1.0)$ & $0(0.0)$ & - & - \\
\hline Endometrial appearance & & & 0.81 & 0 \\
\hline Homogeneous & $67(68.4)$ & $8(66.7)$ & - & - \\
\hline Heterogeneous & $20(20.4)$ & $2(16.7)$ & - & - \\
\hline Trilaminar & $10(10.2)$ & $2(16.7)$ & - & - \\
\hline Not seen & $1(1.0)$ & $0(0.0)$ & - & - \\
\hline Endometrial echogenicity & & & & 0 \\
\hline Hyperechoic & $61(62.2)$ & $10(83.3)$ & 0.48 & - \\
\hline Isoechoic & $25(25.5)$ & $1(8.3)$ & - & - \\
\hline Hypoechoic & $11(11.2)$ & $1(8.3)$ & - & - \\
\hline Not seen & $1(1.0)$ & $0(0.0)$ & - & - \\
\hline \multicolumn{5}{|l|}{ Other endometrial findings } \\
\hline Fluid filled & $25(25.5)$ & $2(16.7)$ & 0.73 & - \\
\hline Cystic & $0(0.0)$ & $0(0.0)$ & - & - \\
\hline Serpiginous & $6(6.1)$ & $0(0.0)$ & 0.49 & - \\
\hline Other & $1(1.0)$ & $0(0.0)$ & 0.89 & - \\
\hline Not assessed & $36(36.7)$ & $4(33.3)$ & 0.54 & - \\
\hline Not seen & $31(31.6)$ & $6(50.0)$ & 0.17 & - \\
\hline Not sure & $0(0.0)$ & $0(0.0)$ & - & - \\
\hline Final right ovarian volume, $\mathrm{ml}$ & $11.4(9.7)$ & $9.9(3.2)$ & 0.65 & 28 \\
\hline Final left ovarian volume, ml & $11.0(10.8)$ & $12.0(4.0)$ & 0.76 & 22 \\
\hline Total antral follicle count & $14.4(6.4)$ & $12.3(5.3)$ & 0.40 & 44 \\
\hline Impression of cycle phase & & & 1.00 & 0 \\
\hline Follicular & $59(60.2)$ & $8(66.7)$ & - & - \\
\hline Periovulatory & $23(23.5)$ & $3(25.0)$ & - & - \\
\hline Luteal & $15(15.3)$ & $1(8.3)$ & - & - \\
\hline Not sure & $1(1.0)$ & $0(0.0)$ & - & - \\
\hline
\end{tabular}

cervix in OF patients as the cervix is embedded in scar or absent altogether as a result of pressure necrosis. However, even in patients with apparent absence of the cervix by direct visualization, one can ultrasonographically visualize residual cervical tissue. The extent to which this cervix is functionally useful for future fertility and parturition remains uncertain.

Of important note is that we could not visualize the cervix in 8 of the 98 OF patients while we were able to visualize the uterus in all patients. The most likely explanation for nonvisualization of the cervix is that no cervix was present (i.e., the cervix was completely destroyed by the obstructed labor). In this case, their cervical length measurements would be $0 \mathrm{~mm}$, thereby strengthening our findings of shorter cervical lengths in OF patients. If all OF patients with a cervix that could not be visualized were considered to have cervical length of $0 \mathrm{~mm}$, the average cervical length among OF patients would drop from $18.8 \mathrm{~mm}$ to $17.2 \mathrm{~mm}$. However, we cannot rule out nonvisualization secondary to sonographer error or the limitations of our ultrasound equipment. 
TABLE 3: Bivariable associations between Waaldijk classification and final cervical length among $90^{*}$ Malawian women with obstetric fistula.

\begin{tabular}{|c|c|c|c|c|}
\hline Classification & $N$ & Mean (SD) & Mean difference $(95 \% \mathrm{CI})$ & $p$ value \\
\hline \multicolumn{5}{|l|}{ Waaldijk major } \\
\hline I & 28 & $20.0(8.0)$ & 0.00 & - \\
\hline IIAa & 15 & $18.3(6.3)$ & $-1.70(-7.45,4.05)$ & 0.56 \\
\hline $\mathrm{IIAb} / \mathrm{IIBb}$ & 40 & $18.1(10.4)$ & $-1.99(-6.42,2.44)$ & 0.38 \\
\hline Missing & 7 & - & - & - \\
\hline \multicolumn{5}{|l|}{ Waaldijk minor } \\
\hline Small & 26 & $19.8(6.1)$ & 0.00 & - \\
\hline Medium & 26 & $19.6(10.1)$ & $-0.19(-5.14,4.76)$ & 0.94 \\
\hline Large/extensive & 30 & $16.8(9.9)$ & $-2.97(-7.76,1.81)$ & 0.22 \\
\hline Missing & 8 & - & - & - \\
\hline
\end{tabular}

*8 women with obstetric fistula excluded due to missing final cervical length measurement.

This study has a few additional potential limitations. First, the physician who performed the ultrasound examination and surgery was not blinded to the fistula status of the patients. The study design would have prohibited blinding in that ultrasound in OF was performed in the operating theatre. Compromised vaginal anatomy including frequent vaginal stenosis and severe scarring often makes a comprehensive examination impossible prior to anesthesia in OF patients. Also, independent of the site of examination, it would be impossible to blind an examiner to the status of a fistula patient. Another limitation was that the controls represented far fewer patients than the cases. Unfortunately, in this study, it was unavoidable. The fistula center where the study was performed primarily cares for women with OF; hence, there were limited opportunities to recruit women without an OF or a history of prolonged obstructed labor (controls). Despite the highly statistically significant differences we found between cases and controls, the limited number of controls could have resulted in an unrepresentative sample due to unexpected deviants or selection bias related to their presentation to the fistula center for care. We are reassured that the average cervical length in controls was $27.3 \mathrm{~mm}$ which is more consistent if not somewhat shorter than that found in prior studies which measured cervical length in the nonpregnant patient $[12,13]$. Indeed, if there was systematic error in measuring the cervical length towards the lower range, this would have resulted in less statistically significant findings, so this issue is unlikely to have affected our results. Unfortunately, there are no published data for average cervical length in our study population to which we can compare our results.

Despite these limitations, the strengths of this study prevail. There is clear pathophysiologic plausibility to the findings of cervical destruction during the process of obstructed labor. Surgically, we commonly find complete destruction of the bladder base, trigone, anterior vagina, and cervix. It is common to have difficulty finding the cervical os during surgery for OF, or when the cervix is found, there is often no identifiable anterior cervical stroma. A common surgical challenge when repairing these patients is prevention of cervical stenosis and subsequent amenorrhea and hematometra after surgery.

\section{Conclusion}

The implications for the findings of this study are numerous. Up to $70 \%$ of women who experience OF will never have a living child [15]. Determining the role of cervical destruction in the etiology of subsequent infertility, subfertility, or pregnancy loss is an important area of future exploration. There may be opportunities to study the impact of surgical revision of the cervix at the time of OF surgery. Also, OF patients who are found to have a shortened cervix may benefit from cerclage, pessary, hormonal therapy, or other modalities to prevent preterm delivery or early pregnancy loss; however, this would have to be validated with an appropriate prospective study before implementation.

In the rush to cure women and girls with OF from their debilitating urinary incontinence, we have largely neglected other important aspects of women's health care in this vulnerable population. Fecundity and the ability to carry a pregnancy to viability are often as important to our patients as achieving continence. Understanding the role of compromised pelvic anatomy infertility in this population is a critical step to helping these patients realize their reproductive goals.

\section{Disclosure}

A portion of the findings in this study were presented as an oral abstract at XXI FIGO World Congress. The funders of this project had no role in the design or conduct of the study, decision to publish, or preparation of the manuscript.

\section{Conflicts of Interest}

The authors declare that they have no conflicts of interest.

\section{Authors' Contributions}

Jeffrey Wilkinson codeveloped the original concept for this study, performed all of the ultrasound examinations, recorded the data, and participated in the writing and revisions of this manuscript. Angela Bengtson advised on the statistical elements of the study, performed the data analysis, 
assisted in the preparation of the results for the manuscript, and assisted with editing the manuscript. Ennet Chipungu assisted in the conduct of this study, collection of data, and reviewing and editing the manuscript. Rachel Pope reviewed and edited the manuscript in its entirety and contributed to the writing of the introduction and discussion. Both Drs. Chipungu and Pope were included in the final manuscript rather than the presented abstract because they contributed significantly beyond the minimal work that was required to process the abstract in the form of collection of additional data, review of manuscript, and assistance with writing. Bonus Makanani assisted in the protocol development, submission for ethical review, and review and editing of the final manuscript. Margaret Moyo contributed to the design and conduct of the study as well as review of the manuscript. Mwawi Mwale contributed to the design of the study and review of the manuscript. Jennifer Tang assisted in the development of the study concept, was the principal investigator on the American College of Obstetrics and Gynecology grant, and participated in the writing and revisions of this manuscript.

\section{Acknowledgments}

The authors would like to thank William Nundwe, Charity Chisale, Sandra Ngwira, Melvis Longwe, Yohann Chikwatu, Dawn Kopp, Allison Sih, and Julia Ryan for their assistance with implementing this study. This study was supported by a generous grant from the American College of Obstetrics and Gynecology/Gottesfeld-Hohler Research Fellowship in Ultrasound. The authors recognize the support of the Freedom From Fistula Foundation which operates the Fistula Care Centre in Lilongwe, Malawi, where this study was conducted. The authors also recognize One by One, which provides general research support to the Texas Children's/Baylor Obstetric Fistula research team. Support for use of the REDCap database was funded by grant (1UL1TR001111) from the North Carolina Clinical and translational Science Award program of the Division of Research Resources.

\section{References}

[1] D. De Ridder, G. H. Badlani, A. Browning, P. Singh, I. Sombie, and L. L. Wall, "Fistulas in the developing world," in Incontinence, P. Abrams, L. Cardozo, S. Khoury, and A. Wein, Eds., pp. 1419-1458, Health Publications Ltd., Paris, 4th Edition, 2009.

[2] V. E. Aimakhu, "Reproductive functions after the repair of obstetric vesicovaginal fistulae," Fertility and Sterility, vol. 25, no. 7, pp. 586-591, 1974.

[3] J. Emembolu, "The obstetric fistula: factors associated with improved pregnancy outcome after a successful repair," International Journal of Gynaecology and Obstetrics, vol. 39, no. 3, pp. 205-212, 1992.

[4] A. L. Wilson, E. Chipeta, L. Kalilani-Phiri, F. Taulo, and A. O. Tsui, "Fertility and pregnancy outcomes among women with obstetric fistula in rural Malawi," International Journal of Gynaecology and Obstetrics, vol. 113, no. 3, pp. 196-198, 2011.

[5] A. Browning, "Obstetric fistula: clinical considerations in the creation of a new urethra and the management of a subsequent pregnancy," International Journal of Gynaecology and Obstetrics, vol. 99, no. 1, pp. S94-S97, 2007.

[6] B. Hancock and A. Browning, Practical Obstetric Fistula Surgery, Royal Society of Medicine Press, London, UK, 2009.

[7] V. A. Adetiloye and F. O. Dare, "Obstetric fistula: evaluation with ultrasonography," Journal of Ultrasound in Medicine, vol. 19, no. 4, pp. 243-249, 2000.

[8] J. D. Iams, R. L. Goldenberg, P. J. Meis et al., "The length of the cervix and the risk of spontaneous premature delivery. National Institute of Child Health and Human Development Maternal Fetal Medicine Unit Network," New England Journal of Medicine, vol. 334, no. 9, pp. 567-572, 1996.

[9] J. Ludmir, M. B. Landon, S. G. Gabbe, P. Samuels, and M. T. Mennuti, "Management of the diethylstilbestrolexposed pregnant patient: a prospective study," American Journal of Obstetrics and Gynecology, vol. 157, no. 3, pp. 665-669, 1987.

[10] A. Golan, R. Langer, S. Wexler, E. Segev, D. Niv, and M. P. David, "Cervical cerclage-its role in the pregnant anomalous uterus," International Journal of Fertility, vol. 35, no. 3, pp. 164-170, 1990.

[11] D. Reichman, M. R. Laufer, and B. K. Robinson, "Pregnancy outcomes in unicornuate uteri: a review," Fertility and Sterility, vol. 91, no. 5, pp. 1886-1894, 2009.

[12] S. Zemlyn, "The length of the uterine cervix and its significance," Journal of Clinical Ultrasound, vol. 9, no. 6, pp. 267-269, 1981.

[13] A. P. Londero, S. Bertozzi, A. Fruscalzo, L. Driul, and D. Marchesoni, "Ultrasonographic assessment of cervix size and its correlation with female characteristics, pregnancy, BMI, and other anthropometric features," Archives of Gynecology and Obstetrics, vol. 283, no. 3, pp. 545-550, 2011.

[14] P. A. Harris, R. Taylor, R. Thielke, J. Payne, N. Gonzalez, and J. G. Conde, "Research electronic data capture (REDCap)a metadata-driven methodology and workflow process for providing translational research informatics support," Journal of Biomedical Informatics, vol. 42, no. 2, pp. 377-381, 2009.

[15] S. Arrowsmith, C. E. Hamlin, and L. L. Wall, "Obstructed labor injury complex: obstetric fistula formation and the multifaceted morbidity of maternal birth trauma in the developing world," Obstetrical \& Gynecological Survey, vol. 51, no. 9, pp. 568-574, 1996. 


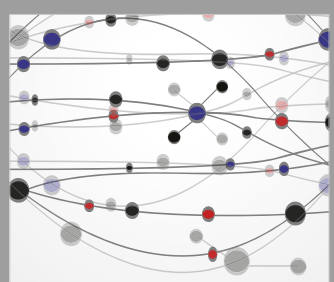

The Scientific World Journal
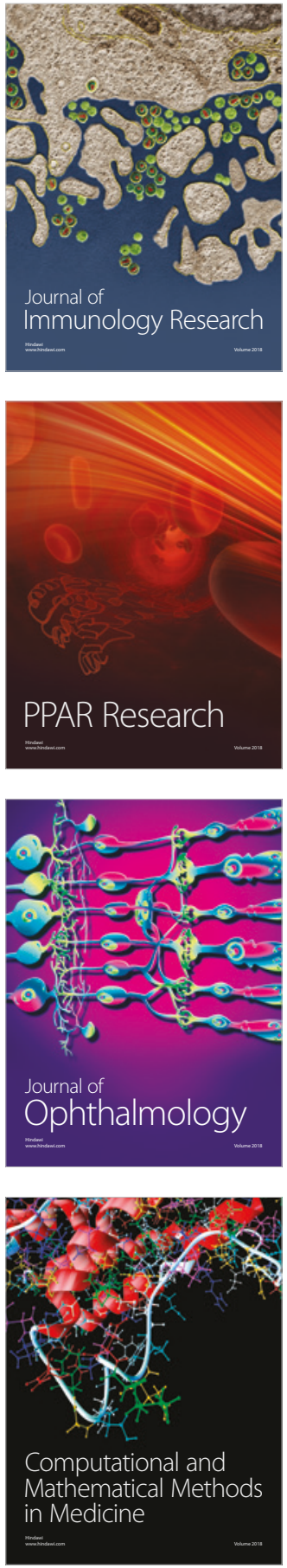

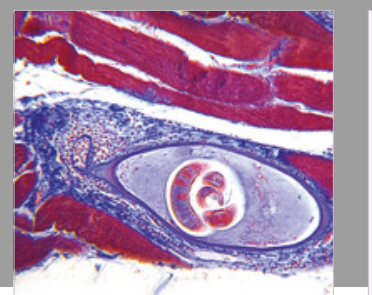

Gastroenterology Research and Practice

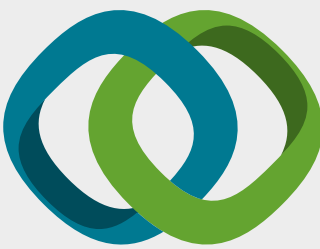

\section{Hindawi}

Submit your manuscripts at

www.hindawi.com
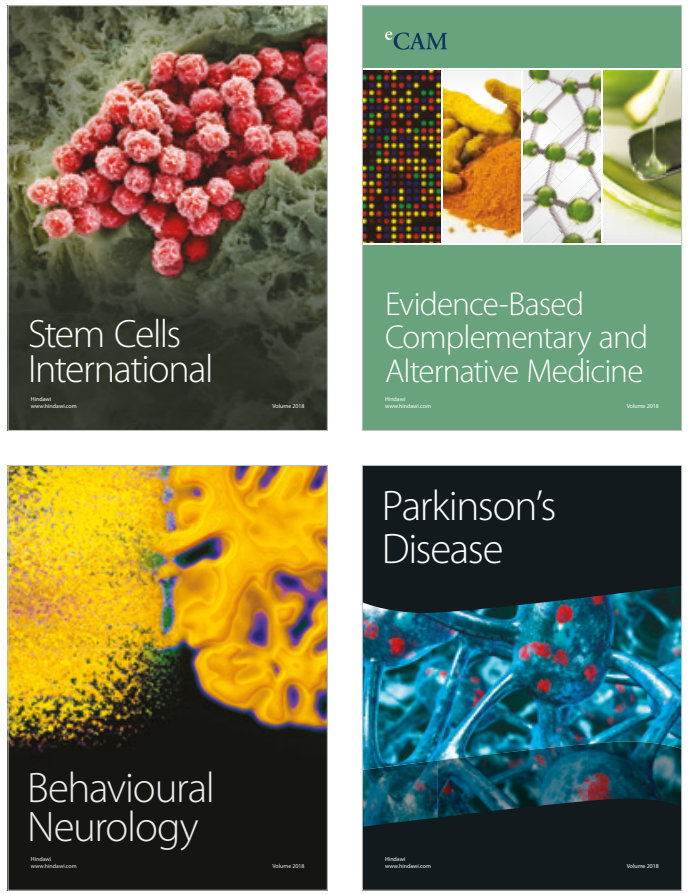

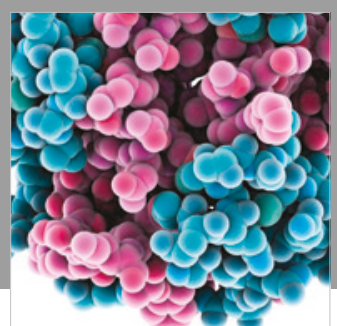

ournal of

Diabetes Research

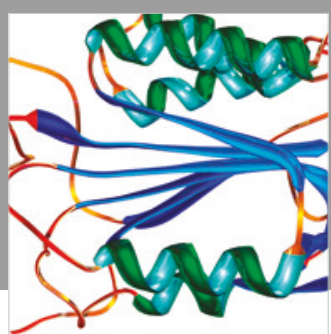

Disease Markers
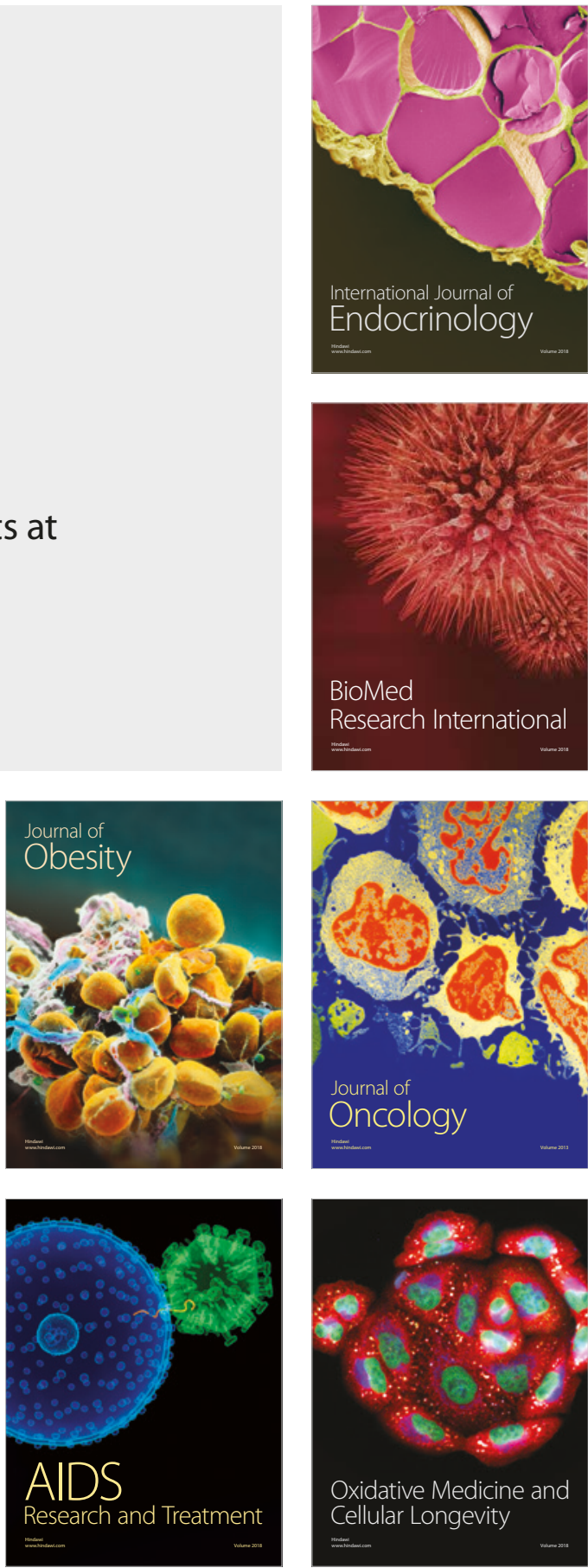